\title{
Bicriteria path problem minimizing the cost and minimizing the number of labels
}

\author{
Marta Pascoal $^{(1,2)}$, M. Eugénia Captivo ${ }^{(3)}$, João Clímaco ${ }^{(2,4)}$, Ana Laranjeira ${ }^{(1)}$ \\ marta@mat.uc.pt, mecaptivo@fc.ul.pt, jclimaco@fe.uc.pt, mat0401@mat.uc.pt \\ (1) Departamento de Matemática da Universidade de Coimbra, \\ Apartado 3008, 3001-454 Coimbra, Portugal \\ (2) Instituto de Engenharia de Sistemas e Computadores - Coimbra \\ Rua Antero de Quental, 199, 3000-033 Coimbra, Portugal \\ (3) Faculdade de Ciências, Universidade de Lisboa \\ Centro de Investigação Operacional \\ Campo Grande, Bloco C6, 1749-016 Lisboa, Portugal \\ (4) Faculdade de Economia da Universidade de Coimbra \\ Avenida Dias da Silva, 165, 3004-512 Coimbra, Portugal
}

\section{Submitted March 2012 Revised December 2012}

\begin{abstract}
We address a bicriterion path problem where each arc is assigned with a cost value and a label (such as a color). The first criterion intends to minimize the total cost of the path (the summation of its arc costs), while the second intends to get the solution with a minimal number of different labels. Since these criteria, in general, are conflicting criteria we develop an algorithm to generate the set of non-dominated paths. Computational experiments are presented and results are discussed.
\end{abstract}

Keywords: Minimal cost, Minimal number of labels, Bicriteria, Shortest path.

MSC classification: 05C85, 90C27, 90C29

\section{Introduction and motivation}

The weight or cost is, probably, the most common objective function in network optimization problems but, besides that, other objective functions are relevant. The number of different colors (or labels) associated with the arcs in a feasible solution is one of those functions, and was introduced by Chang and Leu [3] in the context of determining spanning trees. The goal of the minimal label spanning tree problem (MLSTP) is to find the most uniformly connected spanning subgraph of a network, assuming each edge is associated with a label. Contrarily to the minimal spanning tree problem (MSTP), for which several polynomial algorithms are known [9, 13], [3] proved the NP-hardness of the MLSTP and introduced two heuristic methods, as well as an exponential time algorithm to solve it. Following up that work other approximate algorithms have been developed for the MLSTP and related problems. A literature review on these methods can be found in [7]. Applications of this type of problem include telecommunications, if different labels represent different technologies or operators, and transportation, for instance if referring to different modes of transport [16]. 
A bicriteria version of this problem, the minimal cost-minimal number of labels spanning tree problem (MCLSTP), was studied in [4]. As a first approach to the MCLSTP the computation of all the efficient spanning trees was proposed, by means of an adaptation of the bicriteria algorithm introduced by Clímaco and Martins [5]. This algorithm aims at solving the bicriteria shortest path problem and it is based on the ranking of paths by non-decreasing order of cost. In [4] a similar method was developed that ranks spanning trees and thus computes the efficient spanning trees with respect to cost and number of labels. However, when applied to spanning trees this becomes computationally costly for instances with more than ten colors, even for medium size networks. Therefore, a second approach consists in calculating just one efficient spanning tree for any non-dominated pair of objective values.

In this work we extend the study presented in [4] and address the determination of shortest paths with minimal number of labels. The remainder of the paper is organized as follows. In Section 2 we define the minimal cost - minimal number of labels path problem (MCLPP) and propose different algorithmic approaches. The method that is first proposed generates a set of paths one for each non-dominated pair of objective values. Different implementations of this method are described, and at a second stage reoptimization techniques and cost upper bounds are used in order to enhance the initial method. Section 3 is devoted to the discussion of computational experiments split into two parts, the first involving generic random graphs and random square grids, and the second using random multi-graphs that simulate a transportation network where different means of transportation are distinguished by different arc labels, as well as on a network inspired by the transportation network of Coimbra. The last section presents concluding remarks and future research directions.

\section{The minimal cost and minimal number of labels path problem}

Let $(\mathcal{N}, \mathcal{A})$ be a directed network, where $\mathcal{N}$ denotes the set of $n$ nodes, $\mathcal{A}$ denotes the set of $m$ arcs, and where $\operatorname{arc}(i, j)$ is associated with the cost for using it, $c_{i j} \in \mathbb{R}$, and with one of the $\ell$ possible labels, $l_{i j}$. For convenience each label is represented by an integer in $\{1,2, \ldots, \ell\}$. Let also $c$ and $l$ be two functions such that $c(p)=\sum_{(i, j) \in p} c_{i j}$ denotes the cost of path $p$, and $l(p)$ is the number of different labels in the arcs of path $p$. Given an initial node, $s$, and a terminal node, $t, \mathcal{P}$ denotes the set of paths from $s$ to $t$ in $(\mathcal{N}, \mathcal{A})$.

The goal of the shortest path problem is to determine a minimal cost path between nodes $s$ and $t$ in $(\mathcal{N}, \mathcal{A})$. This problem has been widely studied and, when the network $(\mathcal{N}, \mathcal{A})$ does not contain cycles with a negative cost, it can be solved in polynomial time, for instance, by a labelling algorithm [1]. Because the shortest path problem will be seen as a subproblem of the MCLPP, we assume that the networks satisfy this condition. If the subproblem is to be solved by means of Dijkstra's algorithm then the stronger assumption that the costs are non-negative should be made. When considering $l$ as the objective function we intend to obtain the minimal number of labels path problem, the goal of which is to find a most homogeneous path between two given nodes of $(\mathcal{N}, \mathcal{A})$. Wirth [18] proved this problem is NP-hard. 
The MCLPP consists of determining solutions for the bicriterion problem:

$$
\begin{aligned}
& \min \{c(p): p \in \mathcal{P}\} \\
& \min \{l(p): p \in \mathcal{P}\},
\end{aligned}
$$

that is, paths from $s$ to $t$ which are $\operatorname{good}$ solutions with respect to $c$ and $l$. In general there is a conflict between these functions, and thus there is not a solution that is simultaneously optimal with respect to both. Instead, the literature [15] distinguishes two kinds of solutions, efficient, in the domain of the decision variables, and non-dominated, in the domain of the objective value vectors, defined in the following. Our goal will be to determine the set of all non-dominated solutions.

Since the works by Vincke [17] and Hansen [8] several multicriteria path problems, and in particular bicriteria path problems, have been studied. We mention a few $[2,5,10]$, but for a more complete survey on this subject the reader is referred to $[6,14]$.

A path $p \in \mathcal{P}$ is said to be efficient iff there is no $p^{\prime} \in \mathcal{P}$ such that

$$
c\left(p^{\prime}\right) \leq c(p), \quad l\left(p^{\prime}\right) \leq l(p)
$$

and at least one of the inequalities is strict. When there is $p^{\prime} \in \mathcal{P}$ such that $c\left(p^{\prime}\right) \leq c(p), l\left(p^{\prime}\right) \leq l(p)$ and at least one of these inequalities is strict we say that $\left(c\left(p^{\prime}\right), l\left(p^{\prime}\right)\right)$ dominates $(c(p), l(p))$, for some $p \in \mathcal{P}$. We intend to compute a minimal complete set of paths for the MCLPP, which is a minimal set of efficient paths the images of which cover all non-dominated images. In other words, we look for a set of efficient paths $\overline{\mathcal{P}}$ such that for any two $p, p^{\prime} \in \overline{\mathcal{P}}, p \neq p^{\prime}$, have different images, and for any non-dominated pair $(\bar{c}, \bar{\ell})$ there exists $p \in \overline{\mathcal{P}}$ so that $(c(p), l(p))=(\bar{c}, \bar{\ell})$.

The method proposed here computes a minimal complete set of solutions by calculating the shortest path corresponding to each possible number of labels and checking its dominance. Checking for alternative efficient solutions is still possible, but it is also computationally costly, and usually the added information is not very valuable, thus computing one "representative" efficient solution for each non-dominated pair of objective values is sufficient for most applications.

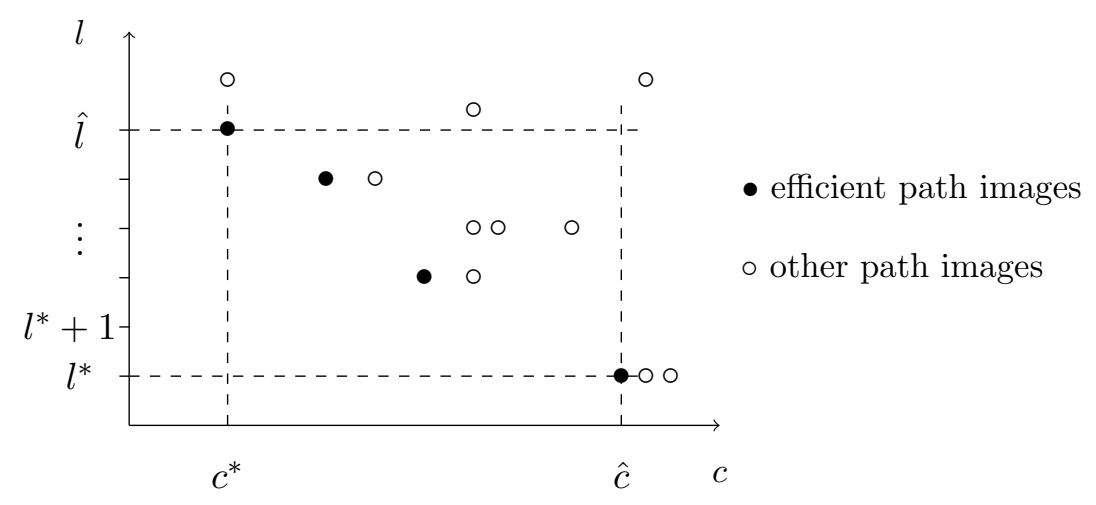

Figure 1: MCLPP solutions

Let $l^{*}\left(c^{*}\right)$ be the minimal number of labels (cost value) of a path, and $\hat{l}(\hat{c})$ be the maximal number of labels (cost value) of an efficient path. These values define the region of the nondominated objective function images, that lies in $\left[c^{*}, \hat{c}\right] \times\left\{l^{*}, \ldots, \hat{l}\right\}$, see Figure 1 . In fact, there are 
no solutions such that $c(p)<c^{*}$ or $l(p)<l^{*}$. Besides, if $c(p) \geq \hat{c}$ and $l(p) \geq l^{*}$ with at least one strict inequality, then $\left(\hat{c}, l^{*}\right)$ dominates $(c(p), l(p))$, and, similarly if $c(p) \geq c^{*}$ and $l(p) \geq \hat{l}$ with at least one strict inequality, then $\left(c^{*}, \hat{l}\right)$ dominates $(c(p), l(p))$.

As mentioned above, we calculate a shortest path corresponding to each number of labels. In fact it is easier to manipulate the network in order to fix the labels a path can contain than to add constraints to fix the path cost. Besides, the range of labels is usually smaller than the range of path costs. For this reason we look for an efficient path with each possible number of labels, as stated in Proposition 1. Before presenting this result we show that the number of labels of all paths from $s$ to $t$ in a network is not necessarily a complete sequence. A counter-example is given in Figure 2, where there are only two paths from $s$ to $t$ in that network,

- $p=\langle s, t\rangle$, with $l(p)=1$, and

- $q=\langle s, 1,2, t\rangle$, with $l(q)=3$,

however none of them has exactly two labels.

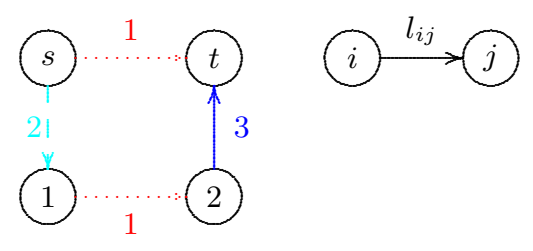

Figure 2: Network with labeled arcs

Proposition 1. For any $k \in\left\{l^{*}, \ldots, \hat{l}\right\}$ :

- either there is no path with $k$ labels,

- or else there is at least a path $p$ from $s$ to $t$ such that $l(p)=k$ and:

- either $p$ is efficient,

- or there is an efficient path $p^{\prime}$ from $s$ to $t$ such that $l\left(p^{\prime}\right)<k$, which dominates all the paths with $k$ labels.

For a fixed number of labels the shortest path dominates other paths with a greater cost. Thus, by Proposition 1 it can be concluded that the set of efficient paths contains the shortest path with $k$ labels, for some of the possible number of labels $k=l^{*}, \ldots, \hat{l}$. Moreover, the application of a single-objective shortest path algorithm with respect to $c$ to $(\mathcal{N}, \mathcal{A})$ provides path $p^{*}$, and thus the value $c^{*}$, the cost of $p^{*}$, and an upper bound on $\hat{l}$, given by the number of labels $l\left(p^{*}\right)$. However, since the minimum label path problem is NP-hard [18], $l^{*}$ cannot be known in polynomial time. Thus, a sequence of constrained shortest path problems in networks with a specified number of labels, ranging between 1 and $l\left(p^{*}\right)$, are solved.

Some notation is now introduced. Let $l(E)=\left\{l_{i j}:(i, j) \in E\right\}$, for any $E \subseteq \mathcal{A}$. Then, for a fixed $k \in\{1,2, \ldots, \ell\},(\mathcal{N}, \mathcal{A})_{k}$ defines the set of all the networks $\left(\mathcal{N}, A_{k}\right)$, with $A_{k} \subseteq \mathcal{A}$ and 
$\left|l\left(A_{k}\right)\right|=k$. We say that $p$ is a path in $(\mathcal{N}, \mathcal{A})_{k}$ if $p$ is a path in at least one of the networks in $(\mathcal{N}, \mathcal{A})_{k}$. The number of labels in every set $(\mathcal{N}, \mathcal{A})_{k}$ is bounded, therefore all its paths $p$ satisfy $l(p) \leq k$. Denoting by $p_{k}$ the shortest path from $s$ to $t$ in $(\mathcal{N}, \mathcal{A})_{k}$, as mentioned before the set $\left\{\left(c\left(p_{k}\right), l\left(p_{k}\right)\right): k=l^{*}, \ldots, \hat{l}\right\}$ contains all the non-dominated objective values of the MCLPP. Each path $p_{k}$ can be computed by examining every subnetwork of $(\mathcal{N}, \mathcal{A})$ in $(\mathcal{N}, \mathcal{A})_{k}$ and, because in general $l^{*}$ is unknown, doing this for $k=1, \ldots, l\left(p^{*}\right)$ provides a set of efficient paths with all the non-dominated objective values. Moreover, Proposition 2 can be used to reduce the number of this type of operations.

Proposition 2. If $l\left(p_{k}\right)=k^{*}<k$, then $p_{k}$ dominates every path $p$ such that $k^{*}<l(p) \leq k$.

Proof. Given a path $p \in \mathcal{P}$ such that $l\left(p_{k}\right)=k^{*}<l(p) \leq k$, both $p$ and $p_{k}$ are paths in $(\mathcal{N}, \mathcal{A})_{k}$ so, by definition of $p_{k}, c\left(p_{k}\right) \leq c(p)$, which means that $p_{k}$ dominates $p$.

This result allows to skip the shortest path determination for some of the sets $(\mathcal{N}, \mathcal{A})_{k}$, whenever $l\left(p_{k}\right)<k$, namely for the sets $(\mathcal{N}, \mathcal{A})_{r}, l\left(p_{k}\right) \leq r \leq k$. This is particularly useful if $k$ is taken by decreasing order, considering the sequence $\left\{(\mathcal{N}, \mathcal{A})_{k}\right\}_{k=l\left(p^{*}\right)}^{1}$. Algorithm 1, together with Procedure 1, summarize this method for computing a set of paths with all the non-dominated objective function values.

Algorithm 1. Finding a minimal complete set for the MCLPP

// Input: network $(\mathcal{N}, \mathcal{A})$ with arc costs and labels; nodes $s$ and $t$

// Output: $\bar{P}$, a minimal complete set of paths from $s$ to $t$ with respect to $c$ and $l$

$/ / P$ is a set that contains possible efficient paths

$01 p^{*} \leftarrow$ shortest path from $s$ to $t$ in $(\mathcal{N}, \mathcal{A})$

$02 P \leftarrow\left\{p^{*}\right\}$

$03 k \leftarrow l\left(p^{*}\right)-1$

04 While $k \geq 1$ Do

$05 \quad p_{k} \leftarrow$ Finding the shortest path from $s$ to $t$ in $(\mathcal{N}, \mathcal{A})_{k}$, as in Procedure 1

06 If $p_{k}$ exists Then $P \leftarrow P \cup\left\{p_{k}\right\}$

$07 \quad k \leftarrow \min \left\{l\left(p_{k}\right), k\right\}-1$

08 EndWhile

$09 \bar{P} \leftarrow P$ after removal of the dominated paths

Note that $(\mathcal{N}, \mathcal{A})$ is the only network in set $(\mathcal{N}, \mathcal{A})_{\ell}$, however the number of labels of $p_{\ell}$, i.e. $p^{*}$, is not necessarily $\hat{l}$, but rather an upper bound on that value. The value $\hat{l}$ coincides with the number of labels of the lexicographically minimal path, therefore either $\hat{l}=l\left(p_{\ell}\right)$, and thus there are no paths with cost $c^{*}$ and less than $\hat{l}$ labels, or there is another path $\bar{p}$ such that $l(\bar{p})=\hat{l}<l\left(p_{\ell}\right)$, and thus $c(\bar{p})=c^{*}$ (otherwise $p_{\ell}$ dominates $\bar{p}$ ). Nevertheless $\hat{l}$ is obtained after a path with a cost greater than $c^{*}$ is computed or when the algorithm exits the While loop, which means that there are no more paths to be found. Moreover, instead of filtering the dominated paths that are stored in $P$ only at the end of the algorithm, these paths can be filtered within the loop. The constrained problem at line 05 is solved by computing the shortest path problem from $s$ to $t$ in every subnetwork of $(\mathcal{N}, \mathcal{A})_{k}$, as outlined in Procedure 1. 
Procedure 1. Finding the shortest path from $s$ to $t$ in $(\mathcal{N}, \mathcal{A})_{k}$

// Input: network $(\mathcal{N}, \mathcal{A})_{k}$ with arc costs and labels;

nodes $s$ and $t$

// Output: $p_{k}$, a shortest path from $s$ to $t$ in the set $(\mathcal{N}, \mathcal{A})_{k}$

01 BestCost $\leftarrow+\infty$

02 For every $\left(\mathcal{N}, A_{k}\right)$ subnetwork of $(\mathcal{N}, \mathcal{A})_{k}$ Do

$03 \quad p \leftarrow$ shortest path from $s$ to $t$ in $\left(\mathcal{N}, A_{k}\right)$

04 If $c(p)<$ BestCost Then

$05 \quad$ BestCost $\leftarrow c(p)$

$06 \quad p_{k} \leftarrow p$

$07 \quad$ EndIf

08 EndFor

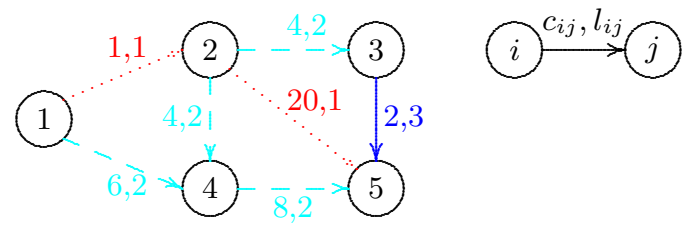

Figure 3: Network $(\mathcal{N}, \mathcal{A})$

As an illustration of Algorithm 1 we consider its application to the network represented in Figure 3 with $s=1$ and $t=5$, which is split into three steps corresponding to the sets of networks $(\mathcal{N}, \mathcal{A})_{k}$, with $k=3,2,1$. Table 1 shows a scheme of the paths that are calculated throughout the procedure.

Table 1: Paths calculated by Procedure 1 applied to the network in Figure 3

\begin{tabular}{cllllll} 
Step & $(\mathcal{N}, \mathcal{A})_{k}$ & $l\left(\mathcal{A}_{k}\right)$ & $p$ & $(c(p), l(p))$ & $p_{k}$ & $P$ \\
\hline 1 & $(\mathcal{N}, \mathcal{A})_{3}$ & $\{1,2,3\}$ & $\langle 1,2,3,5\rangle$ & $(7,3)$ & $\langle 1,2,3,5\rangle$ & $\{\langle 1,2,3,5\rangle\}$ \\
\hline 2 & $(\mathcal{N}, \mathcal{A})_{2}$ & $\{1,2\}$ & $\langle 1,2,4,5\rangle$ & $(13,2)$ & & \\
& & $\{1,3\}$ & $\langle 1,2,5\rangle$ & $(21,1)$ & & \\
& & $\{2,3\}$ & $\langle 1,4,5\rangle$ & $(14,1)$ & $\langle 1,2,4,5\rangle$ & $\{\langle 1,2,3,5\rangle,\langle 1,2,4,5\rangle\}$ \\
\hline 3 & $(\mathcal{N}, \mathcal{A})_{1}$ & $\{1\}$ & $\langle 1,2,5\rangle$ & $(21,1)$ & & \\
& & $\{2\}$ & $\langle 1,4,5\rangle$ & $(14,1)$ & $\langle 1,4,5\rangle$ & \\
& & $\{3\}$ & - & - & & $\{\langle 1,2,3,5\rangle,\langle 1,2,4,5\rangle,\langle 1,4,5\rangle\}$
\end{tabular}

In Procedure 1 it is assumed that for each number of labels $k$ all the subnetworks of $(\mathcal{N}, \mathcal{A})$ with their set of arcs restricted to contain exactly $k$ labels are considered. One way to implement this is to enumerate all the $k$ elements combinations of the $\ell$ labels, and mark all the arcs labeled with other values as non-available. If done by increasing order of the number of labels, the construction of these combinations can be aided by a search tree, with a root with no labels associated, and such that each child node contains a new label that is added to its ancestors' labels. Figure 4 shows an example of such a tree, if the arc labels are 1, 2 and 3.

Given a total number of $\ell$ labels, retrieving all the paths from the root to a level $k$ of the search tree provides all the combinations of those labels with $k$ elements. If the tree is constructed following a breadth first search (BFS) policy, each of its levels, for instance $k$, can support the shortest path 


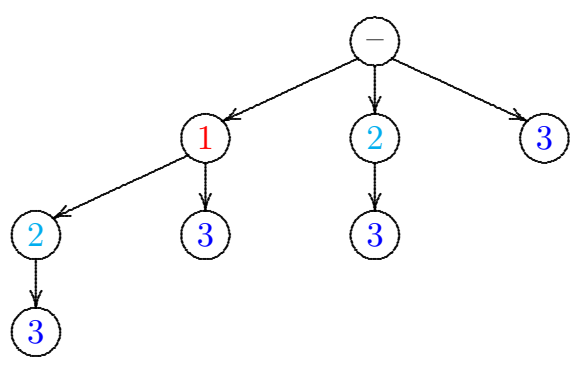

Figure 4: Search tree of a network with the labels 1, 2 and 3.

determination in $(\mathcal{N}, \mathcal{A})_{k}$. Algorithm 2 shows the pseudo-code of an alternative to Algorithm 1 that considers an increasing sequence of the number of labels provided by a BFS tree, the nodes of which result from adding the arcs with a certain label to the shortest path associated with its father. In order to implement the BFS, the set $X$ manages the tree nodes by being manipulated in a first in first out (FIFO) manner. The nodes in each level correspond to all combinations of $k$ elements of $\{1, \ldots, \ell\}$, as depicted in Figure 4.

Algorithm 2. Finding a minimal complete set for the MCLPP with a BFS tree

// Input: network $(\mathcal{N}, \mathcal{A})$ with arc costs and labels; nodes $s$ and $t$

// Output: $\bar{P}$, a minimal complete set of paths from $s$ to $t$ with respect to $c$ and $l$

$/ / P$ is a set that contains possible efficient paths

$/ / C$ is an auxiliary set manipulated in order to obtain all the label combinations

$01 p^{*} \leftarrow$ shortest path in $(\mathcal{N}, \mathcal{A})$

$02 X \leftarrow\{\emptyset\}$

$03 P \leftarrow\left\{p^{*}\right\}$

$04 x \leftarrow 0$

05 While $x \leq l\left(p^{*}\right)$ and $X \neq \emptyset$ Do

$06 \quad C \leftarrow$ first element in $X$

$07 \quad X \leftarrow X-\{C\}$

$08 \quad x \leftarrow|C|$

$09 y \leftarrow$ greatest label in $C$ (or 0 if $C=\emptyset$ )

10 For $k=y+1, \ldots, \ell$ Do

11 Insert $C \cup\{k\}$ at the end of $X$

$12 \quad A^{\prime} \leftarrow\{\operatorname{arcs}$ in $\mathcal{A}$ with a label in $C \cup\{k\}\}$

$13 \quad p \leftarrow$ shortest path in $\left(\mathcal{N}, A^{\prime}\right)$

14 If $p$ exists Then $P \leftarrow P \cup\{p\}$

15 EndFor

16 EndWhile

$17 \bar{P} \leftarrow P$ after removal of the dominated paths

The previous methods compute the shortest path in every subnetwork of $(\mathcal{N}, \mathcal{A})$ obtained by making combinations of the original $\ell$ labels, which means that the number of operations performed by these methods is proportional to $\sum_{k=1}^{\ell}\left(\begin{array}{l}\ell \\ k\end{array}\right)$, and thus it increases quickly with $\ell$. However, two aspects can be taken into account when aiming to reduce that number.

Reoptimization of paths Many of the shortest path problems that have to be solved are similar, only the set of arcs may change by including or excluding arcs with a certain label at a time. The use of this information depends on the algorithm implementation. Although this is not clear for 
Algorithm 1, it is easy to see that, when using Algorithm 2, the problem, and the path, associated with a node in the search tree differs from its ancestor because the arcs with a new label can be used and become part of the solution. This allows to replace many of the shortest path problems with the reoptimization of a shortest path after the inclusion of a set of new arcs in the network. The resulting method is summarized in Algorithm 3.

Algorithm 3. Finding a minimal complete set for the MCLPP with a BFS tree and path reoptimization

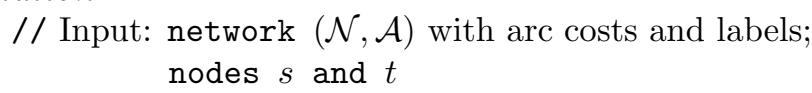

$18 \bar{P} \leftarrow P$ after removal of the dominated paths

Algorithm 3 uses Procedure 2 as the reoptimization method. This is based on a label correcting approach - see [1] - and assumes that a shortest path tree rooted at $s$ is already known as the solution to a previous subproblem. The procedure then works in two phases. First the arcs labeled with the new inserted color are analyzed, and if they allow the improvement of the label of a node this node is inserted back to the list of temporary node labels. Second the labelling procedure proceeds as usual, trying to improve the current paths and using the new color as well as the previous till all node labels are definite.

Procedure 2. Shortest path obtained from the insertion of a set of arcs with label $k$ in a path $p$ // Input: network $\left(\mathcal{N}, A^{\prime}\right)$;

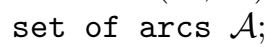




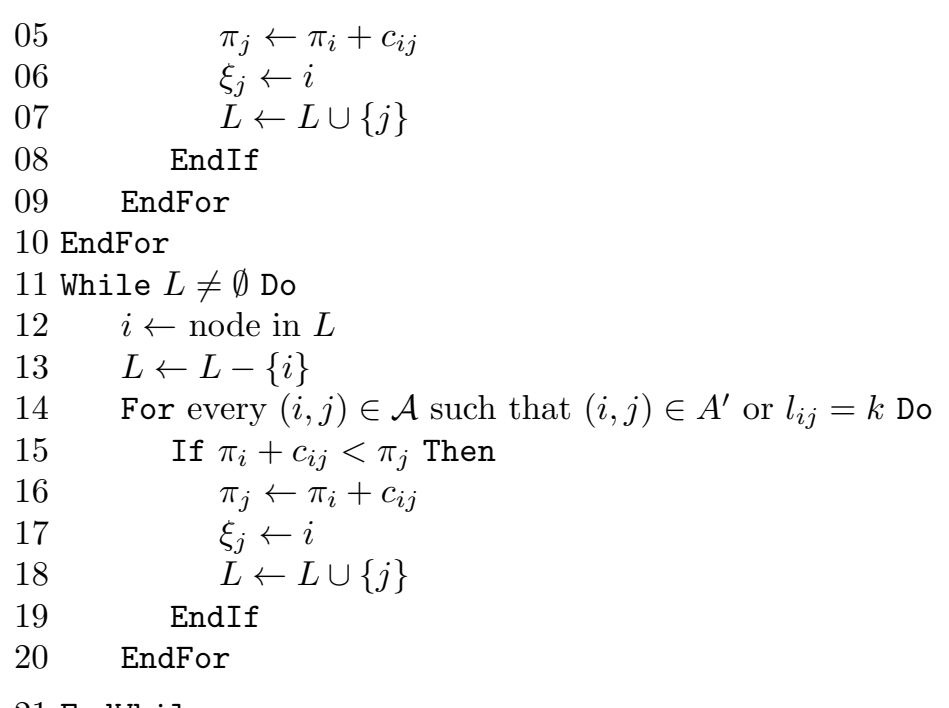

21 EndWhile

\subsection{Enhancing paths computation}

In the shortest path problems that are solved, many repeated paths may be obtained. For instance, in the example above the path $\langle 1,4,5\rangle$ is calculated twice, first as the shortest path in one of the networks in $(\mathcal{N}, \mathcal{A})_{2}$, and second in one of the networks in $(\mathcal{N}, \mathcal{A})_{1}$. However, because that path is not optimal for $(\mathcal{N}, \mathcal{A})_{2}$ it is only stored the second time it is computed. Proposition 3 is a simple result which shows that this information can be managed in order to limit the labels produced in intermediate shortest path problems. According to this result the cost of any path computed at a certain step of the algorithm can be used as a cost upper bound of forthcoming permanent labels. In the case above, in step 2 the objective values of $\langle 1,4,5\rangle$ are calculated $(14,1)$, therefore in step 3 the path $\langle 1,2,5\rangle$, with image $(21,1)$, does not have to be computed till the end.

Proposition 3. Let $p \in \mathcal{P}$, then $c(p)$ is an upper bound on the cost of the efficient paths with $k$ labels, for any $k \geq l(p)$.

Proof. Let $q$ be an efficient path with $l(q)=k \geq l(p)$ and assume, by contradiction, that $c(q)>c(p)$. Then $p$ dominates $q$, and $q$ cannot be efficient.

Corollary 1. Let $p^{*}$ denote the shortest path in $(\mathcal{N}, \mathcal{A})$, and $p_{k}$ denote the shortest path in $(\mathcal{N}, \mathcal{A})_{k}$, that is, a path such that $c\left(p_{k}\right) \leq c(p)$ for any $p$ which satisfies $l(p) \leq k, k=l\left(p^{*}\right), \ldots, 1$. Then $\left\{\left(c\left(p_{k}\right), l\left(p_{k}\right)\right): k=l\left(p^{*}\right), \ldots, 1\right\}$ contains all the non-dominated pairs of objective values.

Proof. Let $\bar{p}$ be an efficient path and assume $(l(\bar{p}), c(\bar{p})) \notin\left\{\left(c\left(p_{k}\right), l\left(p_{k}\right)\right): k=l\left(p^{*}\right), \ldots, 1\right\}$. Two cases should be considered,

1. $l(\bar{p})=l\left(p_{k}\right)$ for some $k=l\left(p^{*}\right), \ldots, 1$,

2. $l(\bar{p}) \neq l\left(p_{k}\right)$ for any $k=l\left(p^{*}\right), \ldots, 1$.

In case 1. $c(\bar{p}) \geq c\left(p_{k}\right)$, because by definition $p_{k}$ is the shortest path in $(\mathcal{N}, \mathcal{A})_{k}$, and $l(\bar{p})=l\left(p_{k}\right)$. This means that $p_{k}$ dominates $\bar{p}$ and thus $\bar{p}$ is not efficient. 
In case 2. take $k=l(\bar{p})$. Then $p_{k}$ is such that $c\left(p_{k}\right) \leq c(\bar{p})$ and $l\left(p_{k}\right) \leq l(\bar{p})$, with $\left(c\left(p_{k}\right), l\left(p_{k}\right)\right) \neq$ $(c(\bar{p}), l(\bar{p}))$, therefore $p_{k}$ dominates $\bar{p}$ and, again, $\bar{p}$ is not efficient.

These results can be incorporated in the algorithm by storing the best cost of the computed paths with $k$ labels, $k=l\left(p^{*}\right), \ldots, 1$. If the shortest path problems are solved by means of a labelling algorithm [1], the stored upper bounds can be used to prevent the creation of labels with a cost that exceeds the upper bound values. In order to make use of this result, the previous algorithms can store an upper bound of the cost for any number of labels by including an $l\left(p^{*}\right)$-components array, $\operatorname{Cost} U B$, initialized with $\operatorname{Cost} U B_{k}=+\infty, k=1, \ldots, l\left(p^{*}\right)$. Whenever a new path, $p$, is computed in a set of networks with $k$ labels, $\operatorname{Cost} U B$ is updated as

$$
\operatorname{Cost} U B_{r} \leftarrow \min \left\{\operatorname{Cost} U B_{r}, c(p)\right\}, r=l(p), \ldots, k .
$$

Such values can be used to restrict the node labels when computing the shortest path in a network $\left(\mathcal{N}, A_{k}\right), k \in\{1, \ldots, \hat{l}\}$.

Reducing the number of path labels in the original approach reduces the number of calculated paths as well. In particular, considering a fixed number of labels it is less likely that the computation of high cost shortest paths is completed. For instance, the application of Algorithm 1 modified by including the array $\operatorname{Cost} U B$ as described results in the steps in Table 2. After obtaining the path

Table 2: Paths calculated by Algorithm 1 using the array $\operatorname{Cost} U B$ applied to the network in Figure 3

\begin{tabular}{clllllll} 
Step & $\operatorname{CostUB}$ & $(\mathcal{N}, \mathcal{A})_{k}$ & $l\left(\mathcal{A}_{k}\right)$ & $p$ & $(c(p), l(p))$ & $p_{k}$ & $P$ \\
\hline 1 & {$[\infty, \infty, 7]$} & $(\mathcal{N}, \mathcal{A})_{3}$ & $\{1,2,3\}$ & $\langle 1,2,3,5\rangle$ & $(7,3)$ & $\langle 1,2,3,5\rangle$ & $\{\langle 1,2,3,5\rangle\}$ \\
\hline 2 & {$[\infty, 13,7]$} & $(\mathcal{N}, \mathcal{A})_{2}$ & $\{1,2\}$ & $\langle 1,2,4,5\rangle$ & $(13,2)$ & $\langle 1,2,4,5\rangle$ & $\{\langle 1,2,3,5\rangle,\langle 1,2,4,5\rangle$ \\
\hline 3 & {$[21,13,7]$} & $(\mathcal{N}, \mathcal{A})_{1}$ & $\{1\}$ & $\langle 1,2,5\rangle$ & $(21,1)$ & & \\
& {$[14,13,7]$} & $(\mathcal{N}, \mathcal{A})_{2}$ & $\{2\}$ & $\langle 1,4,5\rangle$ & $(14,1)$ & $\langle 1,4,5\rangle$ & \\
& & & $\{3\}$ & - & - & & $\{\langle 1,2,3,5\rangle,\langle 1,2,4,5\rangle,\langle 1,4,5\rangle\}$
\end{tabular}

$p=\langle 1,2,4,5\rangle$ such that $c(p)=13$ and $l(p)=2$ for $(\mathcal{N}, \mathcal{A})_{2}$, the paths $\langle 1,2,5\rangle$ and $\langle 1,4,5\rangle$ are no longer generated in step 2 . However, because they have only one label they are still generated along step 3. Generally speaking, after a path $p^{*}$ is obtained in $(\mathcal{N}, \mathcal{A})_{k}$ no other path $q$ in the same set of networks and such that $c(q) \geq c\left(p^{*}\right)$ is considered, regardless of its number of labels. However, such a path $q$ can be efficient if $l(q)<l\left(p^{*}\right)$, and thus $q$ is still eligible for some set of networks $(\mathcal{N}, \mathcal{A})_{r}$, with $l(q) \leq r<l\left(p^{*}\right)$.

In terms of implementation let us assume a labelling algorithm is used to solve each shortest path problem, and let $\pi_{i}$ denote the cost of a path from $s$ to $i$ throughout that algorithm, for any $i \in \mathcal{N}$. In a regular run of a labelling algorithm the label of a node $j$ is updated if there is a node $i$ such that $(i, j) \in \mathcal{A}$ and $\pi_{i}+c_{i j}<\pi_{j}$. When considering a network in $(\mathcal{N}, \mathcal{A})_{k}, k=1, \ldots, \hat{l}$, and taking the upper bound on the paths cost into account the value $\pi_{j}$ is only updated if

$$
\pi_{i}+c_{i j}<\pi_{j} \text { and } \pi_{i}+c_{i j} \leq \operatorname{Cost} U B_{k}
$$


is satisfied. When applying Procedure 1 several paths $p$ are determined until $p_{k}$ is known, so their costs can be used to update $\operatorname{Cost} U B_{l(p)}$ if $c(p)<\operatorname{Cost} U B_{l(p)}$. In that case the path $p$ should be stored, besides paths $p_{k}$.

Tightening upper bounds The above path costs can still be strengthened if combined with information about the cost of the best paths from intermediate nodes to $t$. Let us assume that the shortest path from any node $i \in \mathcal{N}$ to $t$ in $(\mathcal{N}, \mathcal{A})$ is obtained at an early stage of the algorithm (which can be done by means of a labelling method [1]). Let $\mathcal{T}_{t}$ denote the tree, rooted at $t$, containing all those paths, and $\pi_{i}^{t}$ denote the cost of the path from $i$ to $t$ in that tree. $\mathcal{T}_{t}$ provides lower bounds for the cost of paths, which can be used to prevent useless node labels by restricting some node $j$ 's labelling to the cases which satisfy

$$
\pi_{i}+c_{i j}<\pi_{j} \text { and } \pi_{i}+c_{i j}+\pi_{j}^{t} \leq \operatorname{Cost} U B_{k}
$$

on some network in $(\mathcal{N}, \mathcal{A})_{k}, k=1, \ldots, \hat{l}$. In order to be able to use this cost lower bounds and to discard some node labels without penalizing too much the number of performed operations, we propose $\mathcal{T}_{t}$ is calculated only once, prior to the While-Do loop in Algorithm 3, with respect to the original network $(\mathcal{N}, \mathcal{A})$.

\section{Computational experiments}

Empirical experiments were made in order to evaluate some implementations of the presented methods. The tests ran on a Dual Core AMD Opteron at $2 \mathrm{GHz}$, with $4 \mathrm{~Gb}$ of RAM. The following codes, written in $\mathrm{C}$ language and compiled with the option $-\mathbf{0 3}$, were implemented:

- V1: a version of Algorithm 1. V1 constructs iteratively all the combinations with $k$ elements of the $\ell$ labels, for given values of $\ell$ and $k$;

- V2: an improved version of V1 enhanced with comparing the computed path costs to the previously determined;

- V3: an improved version of V2 enhanced with the information on the minimum cost of a path from any node to $t$, computed once at the beginning of the algorithm;

- BFS1: a version of Algorithm 2, using a BFS tree. The combinations with $k$ elements of $\ell$ labels are obtained by adding one label at a time to a previous combination with less elements;

- BFS2: a variant of BFS1, reoptimizing shortest paths in every level of the search tree, as in Algorithm 3.

The single-objective shortest path problems were solved by a label correcting algorithm with the set of temporary labels managed as a FIFO list. 
Random graphs In a first set of tests we considered thirty randomly generated graph instances of each dimension, with $n=100,200, \ldots, 1000, m=5 n, 10 n, 20 n$, and $\ell=5,10,20$. The source and the destination nodes, $s$ and $t$, were chosen randomly, the arc costs and arc labels were uniformly generated in $\{1,2, \ldots, 100\}$ and in $\{1, \ldots, \ell\}$, respectively. It was assumed that there are no parallel arcs nor self-loops.

Table 3: Mean value of $|\bar{P}|$ in random graphs

\begin{tabular}{cccccccccc}
$m$ & \multicolumn{3}{c}{$n \times 5$} & \multicolumn{3}{c}{$n \times 10$} & \multicolumn{3}{c}{$n \times 20$} \\
\hline$\ell$ & 5 & 10 & 20 & 5 & 10 & 20 & 5 & 10 & 20 \\
\hline$n=100$ & 2.667 & 2.933 & 3.000 & 2.733 & 2.867 & 2.800 & 3.067 & 3.600 & 3.667 \\
$n=200$ & 2.333 & 2.667 & 2.467 & 3.200 & 3.533 & 3.933 & 3.200 & 3.667 & 3.333 \\
$n=300$ & 2.800 & 3.133 & 2.733 & 3.400 & 3.600 & 3.600 & 3.267 & 4.067 & 3.733 \\
$n=400$ & 2.267 & 2.600 & 2.533 & 3.600 & 4.000 & 4.200 & 3.733 & 4.933 & 4.667 \\
$n=500$ & 2.733 & 2.867 & 2.867 & 3.467 & 3.667 & 4.067 & 3.133 & 3.867 & 4.467 \\
$n=600$ & 2.933 & 3.933 & 3.933 & 3.667 & 4.467 & 4.933 & 3.400 & 4.333 & 4.133 \\
$n=700$ & 2.333 & 3.133 & 2.867 & 4.067 & 4.400 & 4.933 & 3.667 & 4.867 & 4.667 \\
$n=800$ & 2.733 & 3.000 & 2.467 & 3.267 & 3.533 & 3.733 & 3.733 & 4.733 & 4.867 \\
$n=900$ & 3.067 & 3.400 & 3.133 & 3.400 & 3.467 & 4.067 & 3.800 & 5.200 & 5.333 \\
$n=1000$ & 2.867 & 3.467 & 3.067 & 3.800 & 3.867 & 4.200 & 3.400 & 4.400 & 4.467
\end{tabular}

Let $|\bar{P}|$ denote the cardinality of the minimal complete set of paths for the MCLPP. Table 3 summarizes the mean values of $|\bar{P}|$ obtained for those instances. That number depends on the number of arc labels, $\ell$, which is the maximum value for the cardinality of the minimal complete set for the MCLPP. According to Table 3 the mean cardinality of the minimal complete set for the MCLPP ranged between 2.2 and 5.3, moreover this number usually grows with the average node degree of the graphs.

Table 4 presents running times, in seconds, on this set of tests, whereas Figure 6 shows the ratios with respect to code V1. As expected, the highest increase in the execution times is due to the increase on the number of different labels in the graphs, the times vary from some milliseconds for instances with five labels to tens of seconds for instances with twenty labels. The programs also become slower when the number of nodes or the average node degree is larger, although this performance is not always stable.

The graphics show that, almost always the versions using the search tree (BFS1 and BFS2) are worse than V2 and V3, and that the gap is bigger when $\ell$ is bigger too. Moreover, the worst of the codes based on the search tree, BFS1, is only better than $\mathrm{V} 1$ for small and sparse instances with $m=5 n, 10 n$ and $\ell=5$. BFS2 behaves better than BFS1, it always outperforms $\mathrm{V} 1$ and it also outperforms V2 and V3 for small problems with few nodes and $\ell=5$.

Among the variants of Algorithm 1, V1, V2 and V3, the latter is generally the most efficient and the first the least efficient. The gap is bigger when $\ell$ is bigger too. Both the comparison of the path costs to previous solution costs and the inclusion of upper bounds to limit the number of labels produced during the shortest path computation have a big impact on the program running times, and V2 and V3 CPU times varied from some milliseconds only to 1.100 seconds and 0.331 seconds, respectively. Similarly to what was observed for V1, the performance of V2 and V3 is highly dependent on the number of labels and, in general, it is better for sparser than for denser graphs. 
However, these two variants presented a performance less steady than V1's.

Grid graphs The second set of test instances contains square grid graphs of the form $[1, q] \times$ $[1, q]$, where $q=10,20,30,40,50$. The nodes in the graph correspond to the intersection between horizontal and vertical lines along the grid. The horizontal and vertical lines, together with the diagonal, represent arcs that link each node to all its neighbours in the grid. Such a grid, depicted in Figure 5, has $n=q^{2}$ nodes and $m=4(2 q-1)(q-1)$ arcs. The source node is the bottom border vertex of the grid, whereas the top border is the destination. Like in the case of random instances, arc costs were uniformly generated in $\{1,2, \ldots, 100\}$ and arc labels in $\{1, \ldots, \ell\}$.

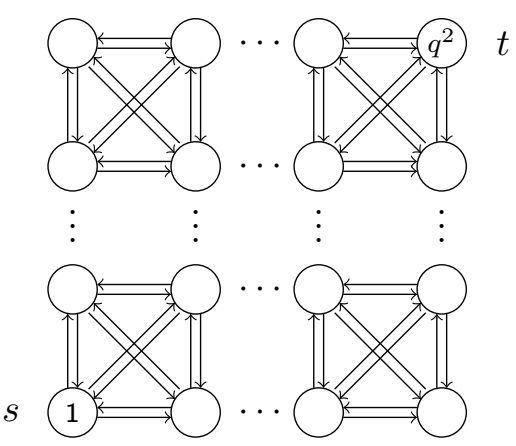

Figure 5: Grid graph

For these instances the mean value of $|\bar{P}|$ is greater than for the random. The mean value varies between 2.1 and 15.7. Therefore, the CPU times are greater for grid graphs too, between a few milliseconds and 2430.503 seconds with the slowest code, BFS1, and between a few milliseconds and 172.201 seconds with the fastest, V3. For this set of tests the performance of V2, V3, BFS1 and BFS2 compared to V1 was worse than for the random set of graphs. Although the relative behavior of the codes is similar, now the versions based on a search tree are worse or equivalent to V1, whereas V2 is slightly faster. The advantage of V3 is always clear, although it seems to become smaller when $\ell$ increases. 
Table 4: Mean CPU times (secs) in random graphs

\begin{tabular}{|c|c|c|c|c|c|c|c|c|c|c|}
\hline & $m$ & & $n \times 5$ & & & $n \times 10$ & & & $n \times 2$ & \\
\hline & $\ell$ & 5 & 10 & 20 & 5 & 10 & 20 & 5 & 10 & 20 \\
\hline & $n=100$ & 0.000 & 0.001 & 0.175 & 0.000 & 0.003 & 0.148 & 0.000 & 0.006 & 0.174 \\
\hline & $n=200$ & 0.000 & 0.002 & 0.264 & 0.001 & 0.010 & 2.280 & 0.002 & 0.019 & 1.267 \\
\hline & $n=300$ & 0.000 & 0.005 & 0.583 & 0.002 & 0.016 & 1.485 & 0.003 & 0.044 & 4.226 \\
\hline & $n=400$ & 0.001 & 0.008 & 0.524 & 0.002 & 0.036 & 3.420 & 0.005 & 0.083 & 11.366 \\
\hline V1 & $n=500$ & 0.001 & 0.015 & 1.228 & 0.003 & 0.049 & 3.897 & 0.006 & 0.069 & 8.006 \\
\hline & $n=600$ & 0.002 & 0.023 & 2.208 & 0.004 & 0.070 & 9.434 & 0.007 & 0.101 & 7.441 \\
\hline & $n=700$ & 0.002 & 0.016 & 1.805 & 0.005 & 0.080 & 16.313 & 0.009 & 0.136 & 19.176 \\
\hline & $n=800$ & 0.002 & 0.024 & 1.076 & 0.005 & 0.063 & 8.856 & 0.009 & 0.167 & 25.548 \\
\hline & $n=900$ & 0.002 & 0.028 & 4.371 & 0.006 & 0.068 & 12.575 & 0.011 & 0.220 & 40.890 \\
\hline & $n=1000$ & 0.002 & 0.039 & 3.300 & 0.006 & 0.087 & 14.646 & 0.010 & 0.189 & 28.727 \\
\hline & $n=100$ & 0.000 & 0.000 & 0.039 & 0.000 & 0.000 & 0.021 & 0.000 & 0.001 & 0.007 \\
\hline & $n=200$ & 0.000 & 0.001 & 0.051 & 0.000 & 0.002 & 0.221 & 0.001 & 0.002 & 0.038 \\
\hline & $n=300$ & 0.000 & 0.001 & 0.146 & 0.007 & 0.002 & 0.045 & 0.001 & 0.004 & 0.443 \\
\hline & $n=400$ & 0.001 & 0.001 & 0.053 & 0.001 & 0.003 & 0.169 & 0.001 & 0.009 & 0.802 \\
\hline V2 & $n=500$ & 0.001 & 0.002 & 0.118 & 0.001 & 0.003 & 0.169 & 0.002 & 0.003 & 0.177 \\
\hline & $n=600$ & 0.001 & 0.003 & 0.283 & 0.001 & 0.006 & 0.304 & 0.002 & 0.007 & 0.129 \\
\hline & $n=700$ & 0.001 & 0.0 & 0.188 & 0.001 & 0.009 & 0.479 & 0.002 & 0.016 & 0.711 \\
\hline & $n=800$ & 0 & 0.0 & 0.101 & 0.001 & 0.007 & 0.994 & 0.0 & 0.013 & 0.422 \\
\hline & $n=900$ & 0.001 & 0.005 & 0.312 & 0.002 & 0.005 & 0.380 & 0.003 & 0.016 & 1.100 \\
\hline & $n=1000$ & 0.001 & 0.006 & 0.360 & 0.002 & 0.012 & 0.770 & 0.003 & 0.015 & 0.427 \\
\hline & $n=100$ & 0.00 & 0.0 & 0.018 & 0.000 & 0.000 & 0.004 & 0.00 & 0.000 & 0.005 \\
\hline & $n=200$ & 0.000 & 0.0 & 0.023 & 0.000 & 0.001 & 0.073 & 0.001 & 0.001 & 0.018 \\
\hline & $n=300$ & 0.000 & 0.000 & 0.050 & 0.001 & 0.001 & 0.023 & 0.001 & 0.002 & 0.067 \\
\hline & $n=400$ & 0.000 & 0.001 & 0.035 & 0.001 & 0.001 & 0.072 & 0.001 & 0.004 & 0.149 \\
\hline V3 & $n=500$ & 0.00 & 0.0 & 0.069 & 0.001 & 0.001 & 0.086 & 0.001 & 0.002 & 0.075 \\
\hline & $n=600$ & 0.0 & 0.0 & 0.119 & 0.001 & 0.002 & 0.137 & 0.0 & 0.002 & 0.061 \\
\hline & $n=700$ & 0.001 & 0.001 & 0.093 & 0.001 & 0.003 & 0.193 & 0.002 & 0.008 & 0.262 \\
\hline & $n=800$ & 0.001 & 0.001 & 0.064 & 0.001 & 0.003 & 0.197 & 0.002 & 0.004 & 0.142 \\
\hline & $n=900$ & 0.001 & 0.00 & 0.176 & 0.002 & 0.003 & 0.162 & 0.003 & 0.007 & 0.331 \\
\hline & $n=1000$ & 0.001 & 0.002 & 0.147 & 0.002 & 0.004 & 0.235 & 0.003 & 0.008 & 0.220 \\
\hline & $n=100$ & 0.000 & 0.002 & 0.304 & 0.000 & 0.005 & 0.212 & 0.001 & 0.009 & 0.214 \\
\hline & $n=200$ & 0.000 & 0.006 & 0.566 & 0.001 & 0.023 & 3.518 & 0.002 & 0.039 & 1.953 \\
\hline & $n=300$ & 0.000 & 0.013 & 1.486 & 0.002 & 0.032 & 1.710 & 0.004 & 0.074 & 7.473 \\
\hline & $n=400$ & 0.001 & 0.0 & 0.893 & 0.003 & 0.061 & 6.010 & 0.007 & 0.144 & 22.661 \\
\hline BFS1 & $n=500$ & 0.001 & 0.021 & 1.890 & 0.004 & 0.061 & 5.613 & 0.008 & 0.094 & 10.678 \\
\hline & $n=600$ & 0.003 & 0.044 & 5.128 & 0.006 & 0.121 & 14.209 & 0.012 & 0.171 & 11.224 \\
\hline & $n=700$ & 0.003 & 0.031 & 3.799 & 0.008 & 0.150 & 21.520 & 0.017 & 0.280 & 39.780 \\
\hline & $n=800$ & 0.003 & 0.039 & 1.711 & 0.007 & 0.108 & 26.397 & 0.018 & 0.288 & 28.694 \\
\hline & $n=900$ & 0.003 & 0.0 & 8.240 & 0.008 & 0.111 & 15.311 & 0.021 & 0.391 & 70.836 \\
\hline & $n=1000$ & 0.003 & 0.070 & 7.093 & 0.012 & 0.213 & 30.315 & 0.022 & 0.342 & 39.229 \\
\hline & $n=100$ & 0.000 & 0.000 & 0.061 & 0.000 & 0.000 & 0.030 & 0.000 & 0.001 & 0.017 \\
\hline & $n=200$ & 0.000 & 0.00 & 0.097 & 0.000 & 0.004 & 0.386 & 0.000 & 0.003 & 0.065 \\
\hline & $n=300$ & 0.000 & 0.00 & 0.241 & 0.001 & 0.003 & 0.076 & 0.001 & 0.008 & 0.693 \\
\hline & $n=400$ & 0.000 & 0.002 & 0.078 & 0.001 & 0.005 & 0.262 & 0.002 & 0.014 & 1.307 \\
\hline BFS2 & $n=500$ & 0.000 & 0.002 & 0.162 & 0.001 & 0.005 & 0.291 & 0.002 & 0.006 & 0.398 \\
\hline & $n=600$ & 0.001 & 0.0 & 0.461 & 0.001 & 0.010 & 0.550 & 0.003 & 0.010 & 0.224 \\
\hline & $n=700$ & 0.001 & 0.00 & 0.296 & 0.002 & 0.014 & 0.694 & 0.004 & 0.022 & 1.505 \\
\hline & $n=800$ & 0.001 & 0.00 & 0.137 & 0.002 & 0.010 & 1.797 & 0.005 & 0.021 & 0.773 \\
\hline & $n=900$ & 0.001 & 0.009 & 0.465 & 0.003 & 0.009 & 0.992 & 0.006 & 0.027 & 1.564 \\
\hline & $n=1000$ & 0.001 & 0.009 & 0.720 & 0.003 & 0.018 & 1.389 & 0.006 & 0.021 & 0.522 \\
\hline
\end{tabular}


Table 5: Mean value of $|\bar{P}|$ in grid graphs

\begin{tabular}{cccc}
$\ell$ & 5 & 10 & 20 \\
\hline $10 \times 10$ & 2.100 & 4.633 & 5.400 \\
$20 \times 20$ & 2.133 & 6.400 & 6.233 \\
$30 \times 30$ & 3.000 & 7.933 & 10.367 \\
$40 \times 40$ & 3.000 & 8.000 & 13.200 \\
$50 \times 50$ & 4.000 & 8.000 & 15.700
\end{tabular}

Table 6: Mean CPU times (secs) in grid graphs

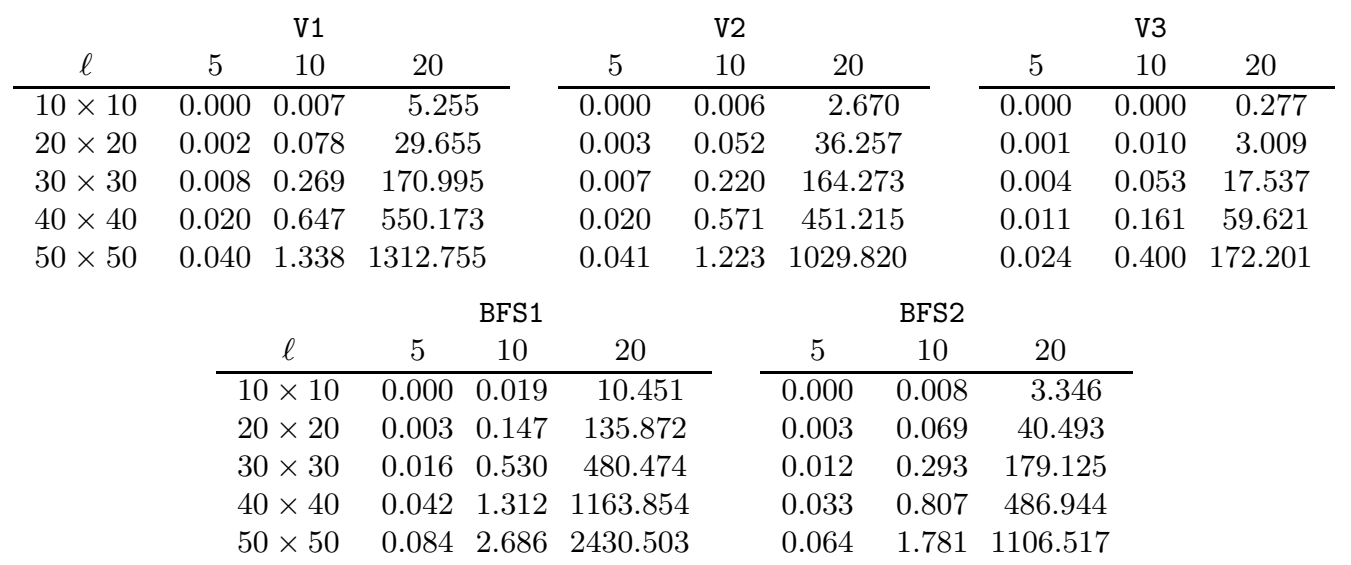



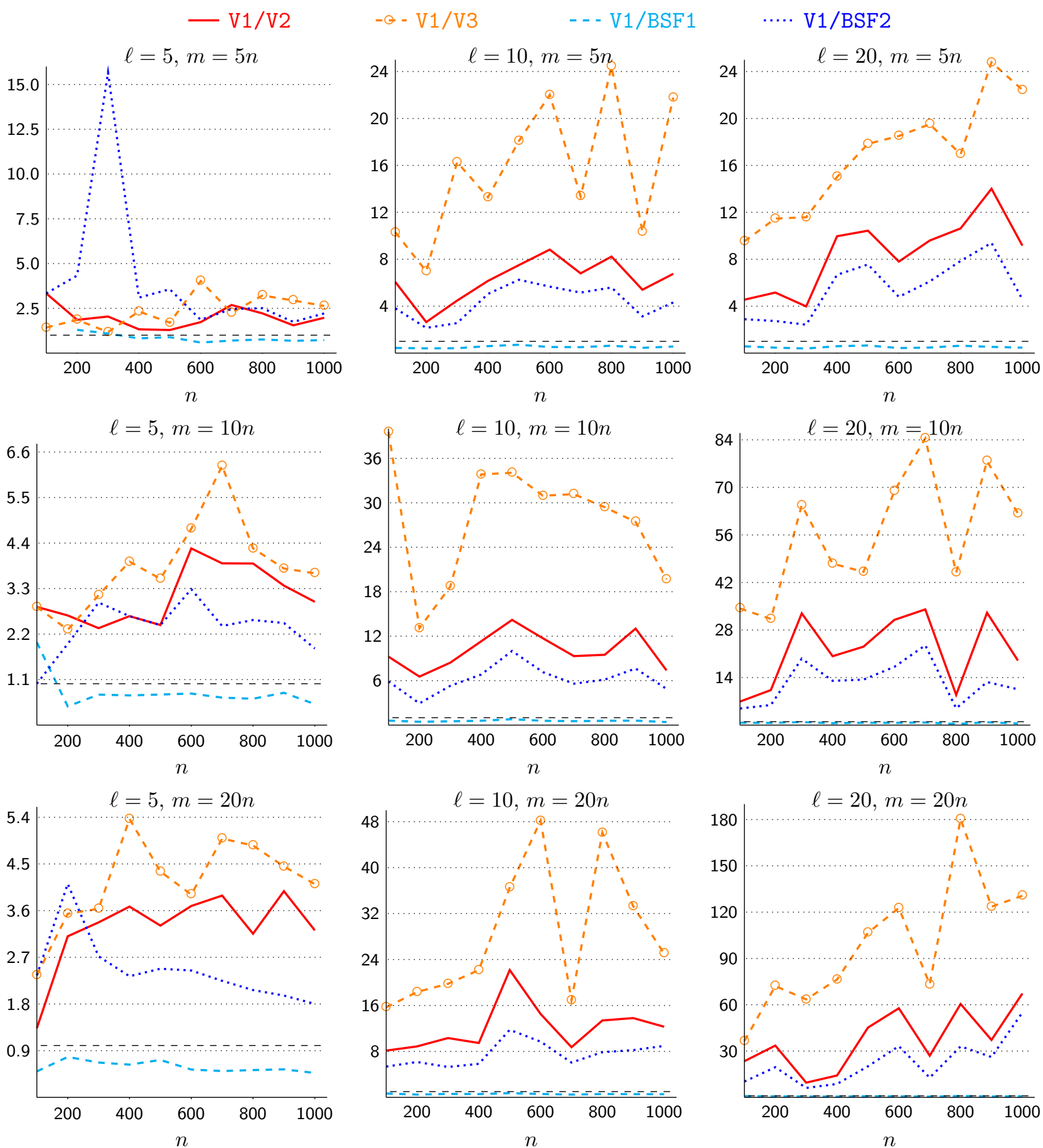

Figure 6: Mean CPU times ratio for random graphs with respect to V1 

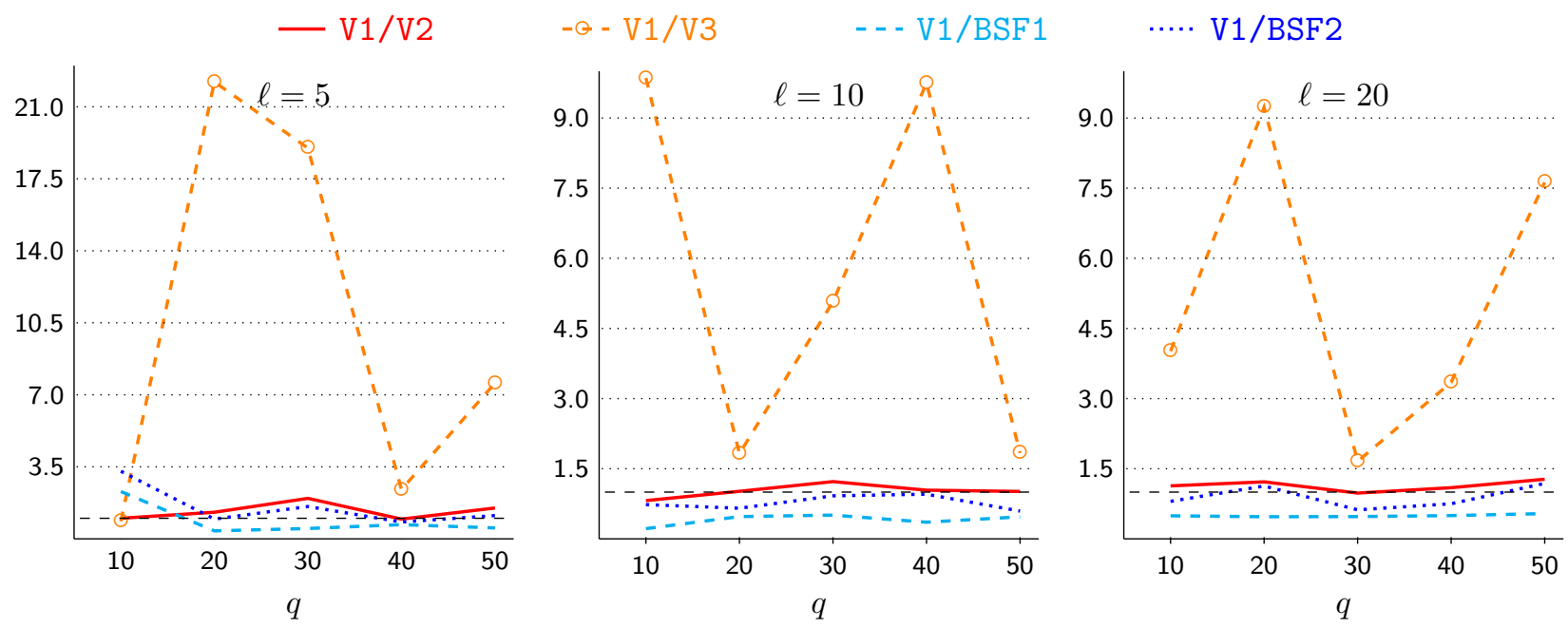

Figure 7: Mean CPU times ratio for grid networks $q \times q$ with respect to V1 
Multi-modal graphs Another set of tests ran on thirty random instances with multi-graphs simulating networks with several means of transportation. The parallel arcs in the graph are identified with different indexes, whereas the transportation modes are distinguished by different labels. The multi-graphs result from overlapping graphs, each corresponding to a mean of transportation. First, instances with three types of transportation $(\ell=3)$ were considered, and second that number was increased to five $(\ell=5)$.

In the case $\ell=3$ it is intended to simulate the following different subnetworks,

- The taxi network, generated as a connected network, $(\mathcal{N}, \mathcal{A})$, with $n=100, \ldots, 1000$ and $m=5 n, 10 n, 20 n$. The costs are integers uniformly obtained in $\{1,2, \ldots, 120\}$. This structure is the most complete of the three and the one that reaches more locations in the network, since it is assumed that taxis are the most flexible transportation.

- The bus network is composed of several routes, of linear or circular format, that is designed by selecting a random number of nodes in $\mathcal{N}$. The number of circuits of each type was twenty, sixty and ninety, whereas the arc costs were obtained as before.

- The subway network is a subtree of $(\mathcal{N}, \mathcal{A})$, containing a random number of nodes. The costs were uniformly generated in $\{1,2, \ldots, 50\}$.

Besides the transportation modes already mentioned for the case $\ell=3$, the case $\ell=5$ also included,

- A cycle-way network, similar to the subway network but with a different number of nodes, and costs in $\{1,2, \ldots, 20\}$.

- A minibus, similar to a linear bus route but with a different number of nodes.

The source and the destination nodes are randomly selected.

Table 7: Mean value of $|\bar{P}|$ and CPU time (secs) of v1, $\ell=3$

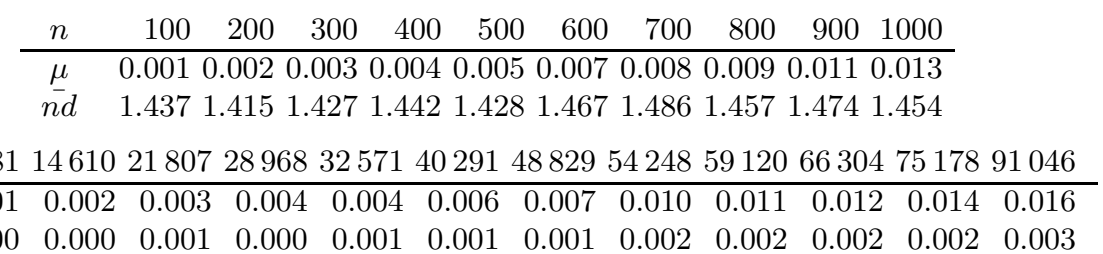

Legend. $\mu$ : Mean CPU times; $\bar{n} d$ : Mean value of $|\bar{P}| ; \bar{m}$ : Mean number of arcs; $\sigma$ : Standard deviation of the CPU times.

The results of these tests are summarized in Tables 7 and 8 for the set of instances with $\ell=3$ and $\ell=5$, respectively. The first part of these tables lists mean values of the running times and of the number of computed solutions for each number of nodes. The values are in accordance with those observed above for instances with 5 labels, the times are very close and the number of solutions is slightly smaller for $\ell=3$ than for $\ell=5$. 
In this set of tests the number of nodes, $n$, and the number of arcs, $m$, in the graphs simulating the taxis network are fixed. However, the sizes of the bus and of the subway networks, as well as the cycle-way and minibus networks (when $\ell=5$ ), vary, so the results are organized into two ways. In the first set of results on Tables 7 and 8 the values obtained are grouped for instances with the same $n$. Then, because the results in terms of CPU times were similar for instances with approximately the same total number of arcs, such values are presented in the second group. The figures reported for the first case are means for the CPU times, $\mu$, and for the number of efficient output solutions, $\overline{n d}$. For the second case the mean running times are calculated over instances with similar numbers of arcs. The standard deviation of the running times in each sample, $\sigma$, is also provided. These standard deviations values are negligible, which confirms the similarity between the running times in instances with approximately the same number of arcs. The mean times increase with $\bar{m}$ and are smaller for the instances with less means of transportation. According to such classification, on average the problems could be solved in less than 16 milliseconds and less than 29 milliseconds, for instances with 3 and 5 means of transportation, respectively.

Table 8: Mean value of $|\bar{P}|$ and CPU time (secs) of v1, $\ell=5$

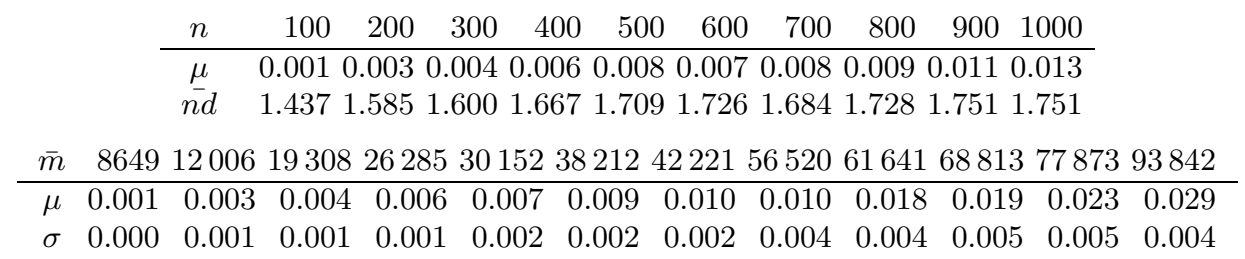

Legend. $\mu$ : Mean CPU times; $\bar{n} d$ : Mean value of $|\bar{P}| ; \bar{m}$ : Mean number of arcs; $\sigma$ : Standard deviation of the CPU times.

The proposed method was also evaluated by tests over data based on part of the city of Coimbra, kindly provided by the INESC-Coimbra research group on urban transportation. This network has 1686 nodes and 8128 directed arcs, and comprises the following $\ell=4$ transportation types: pedestrian, car (taxi), light rail and buses. A travel time is associated with each arc, given by the product of its length and a standard urban speed value for each transportation type. Namely, 4 $\mathrm{km} / \mathrm{h}$ for walking, $15 \mathrm{~km} / \mathrm{h}$ for buses, $17 \mathrm{~km} / \mathrm{h}$ for the light rail, and $30 \mathrm{~km} / \mathrm{h}$ for cars. Applied to this instance the algorithm minimizes the travel time and the number of transportation means, and was tested for ten different origin-destination pairs. The average running time obtained for these problems was 0.002 seconds, to compute an average of 1.100 efficient paths between each origin-destination pair.

\section{Concluding remarks}

Two approaches were proposed for the MCLPP:

- One that relies on the calculation of the shortest path on the set of networks $(\mathcal{N}, \mathcal{A})_{k}$ with $k=\hat{l}, \ldots, 2,1$, together with checking the solutions' dominance. 
- Another that uses BFS to find the shortest path on networks with $k=1,2, \ldots, \hat{l}$, together with reoptimization to derive new paths.

The number of subproblems and operations was reduced by using the data from intermediate subproblems and the pre-computation of $\mathcal{T}_{t}$.

In general the first approach outperformed the one using BFS. The best variant included the use of intermediate computations. Instances with $n=1000$ and $\ell=20$ were solved in less than 0.4 secs. In multi-graphs modelling networks with $\ell=3,5$ transportation modes the efficient paths were computed in less than 0.03 secs. In $50 \times 50$ grid networks the best version CPU times did not exceed 172.3 seconds for $\ell=20$.

Within certain application problems it is desirable to have the least possible number of transitions, namely if switching labels carries some sort of penalization of the solution. For instance, if a passenger wants to travel from a source to a destination and has two options with the same cost and the same number of labels, as depicted in Figure 8, then path $p$, with a single transshipment, is more user convenient than path $q$, with two. This would imply an additional function to minimize the number of transitions. In the near future we intend to study this case.

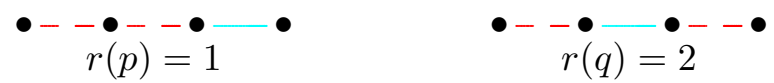

Figure 8: Two paths with the same number of labels

Acknowledgments This work was partially supported by the FCT Portuguese Foundation of Science and Technology (Fundação para a Ciência e a Tecnologia) under projects PEst-C/EEI/UI0308/2011, PEst-OE/MAT/UI0152 and PTDC/EEA-TEL/101884/2008. The authors deeply acknowledge the INESC-Coimbra research group on urban transportation, led by João Coutinho Rodrigues, for providing data about the metropolitan area of Coimbra.

\section{References}

[1] R. K. Ahuja, T. L. Magnanti, and J. B. Orlin. Network Flows: Theory, Algorithms and Applications. Prentice Hall, Englewood Cliffs, NJ, 1993.

[2] C. Bornstein, N. Maculan, M. Pascoal and L. Pinto. Multiobjective combinatorial optimization problems with a cost and several bottleneck objective functions: an algorithm with reoptimization. Computers \& Operations Research, 39:1969-1976, 2012.

[3] R. Chang and S.-J. Leu. The minimum labeling spanning trees. Information Processing Letters, 63(5):277-282, 1997.

[4] J. Clímaco, M. E. Captivo, and M. Pascoal. On the bicriterion - minimal cost/minimal label - spanning tree problem. European Journal of Operational Research, 204:199-205, 2010.

[5] J. Clímaco and E. Martins. A bicriterion shortest path algorithm. European Journal of Operational Research, 11:399-404, 1982. 
[6] J. Clímaco and M. Pascoal. Multicriteria path and tree problems: discussion on exact algorithms and applications. International Transactions in Operational Research, 19:63-98, 2012.

[7] S. Consoli, J. A. Moreno, N. Mladenović, and K. Darby-Dowman. Constructive heuristics for the minimum labelling spanning tree problem: a preliminary comparison. Technical Report DEIOC-4, Universidad de La Laguna, La Laguna, September 2006. (http://hdl.handle.net/2438/504).

[8] P. Hansen. Bicriterion path problems. In Multiple Criteria Decision Making: Theory and Applications, editors: G. Fandel and T. Gal, Lectures Notes in Economics and Mathematical Systems, 1980.

[9] J. Kruskal. On the shortest spanning subtree of a graph and the traveling salesman problem. Proceedings of the American Mathematical Society, 7:48-50, 1956.

[10] M. Iori, S. Martello, and D. Pretolani, An aggregate label setting policy for the multi-objective shortest path problem. European Journal of Operational Research, 207:1489-1496, 2010.

[11] E. Q. V. Martins, M. M. B. Pascoal, and J. L. E. Santos. Deviation algorithms for ranking shortest paths. The International Journal of Foundations of Computer Science, 10(3):247-263, 1999.

[12] M. Pascoal. Implementations and empirical comparison for $K$ shortest loopless path algorithms. In Online Proc. of The Ninth DIMACS Implementation Challenge: The Shortest Path Problem. DIMACS, USA, November 2006.

[13] R. Prim. Shortest connection networks and some generalizations. Bell System Technical Journal, 36:1389-1401, 1957.

[14] A. Raith, and M. Ehrgott. A comparison of solution strategies for biobjective shortest path problems. Computers \& Operations Research, 36:1299-1331, 2009.

[15] R. Steuer. Multiple Criteria Optimization. Theory, Computation and Application. John Wiley, New York, 1986.

[16] R. Van-Nes. Design of multimodal transport networks: A hierarchical approach. Delft University Press, 2002.

[17] P. Vincke. Problèmes multicritères. Cahiers du Centre d'Études de Recherche Opérationelle, 16:425-439, 1974.

[18] H.-C. Wirth. Multicriteria Approximation of Network Design and Network Upgrade Problems. PhD thesis, University of Würzburg, 2001.

[19] J. Y. Yen. Finding the $K$ shortest loopless paths in a network. Management Science, 17:712716, 1971. 